\title{
Pessary cervical and prevention preterm birth based on literature review
}

\begin{abstract}
Preterm birth is the main individual cause of global perinatal morbidity and mortality, cervical insufficiency being one of its causes. One of the hypotheses of cervical insufficiency is that it is due to a mechanical problem; hence one of the approaches is the use of the cervical pessary. We review the most recent literature on the use of the cervical pessary in the prevention of premature birth in single and multiple pregnancies, as well as the indications for its use in different clinical practice guidelines.
\end{abstract}

Keywords: cervical length, cervical pessary, prematurity, preterm birth, short cervix
Volume 4 Issue 4 - 2018

\author{
María del Mar Molina Hita, Laura Revelles \\ Paniza, Susana Ruiz Durán \\ Department of Obstetrics and Gynecology, University Hospital \\ Virgen de las Nieves, Spain
}

\begin{abstract}
Correspondence: Susana Ruiz Durán, Physician Specialist in Obstetrics and Gynaecology, Department of Obstetrics and Gynaecology, University Hospital "Virgen de las Nieves", Granada. Spain, Avd. Divina Pastora 9, Block 13, 4 B. 18012 Granada, Spain, Tel +34666462333.

Email sruizduran@hotmail.com
\end{abstract}

Received: June 20, 2018 | Published: July 27, 2018

\section{Introduction}

Preterm birth is the leading individual cause of global perinatal morbidity and mortality and the leading cause of death and disability in children up to 5 years old in the developed world. ${ }^{1}$ Cervical insufficiency is one of its causes. Its definition is not fixed. It could correlate to a structural abnormality of the biochemical components of the cervical tissue that prevents the inhibition of its function as sphincter. ${ }^{2}$ From the clinical point of view the cervical insufficiency is defined as a cervical painless dilation leading to recurrent pregnancy loss in the second term and premature delivery, depending on the specific characteristics of the cervix, in particular cervical length. ${ }^{3}$

Under normal conditions the length of the cervix is stable between weeks 14 and 28 of gestation. For simple pregnancies from weeks 22 to 24 the distribution is: $15 \mathrm{~mm}$ (percentile 2); $25 \mathrm{~mm}$ (percentile 10); $30 \mathrm{~mm}$ (percentile 50). From weeks 28 to 32 a gradual decrease in cervical length is normal. However, taking all this into account, cervical insufficiency is also defined as a cervical length by transvaginal echography of less than $25 \mathrm{~mm}$ before week 24 of gestation in a woman with a previous spontaneous premature delivery. ${ }^{4}$ The measurement of cervical length in mid pregnancy has been shown to be able to predict accurately preterm delivery. The limitation of screening for the shortened cervix is its low prevalence ranging from 0.9 to $2.3 \%$ generally, according to the population and the limit used (under 15, 20 or $25 \mathrm{~mm}$ ). Transvaginal ultrasound is the method of choice to identify patients at higher risk of spontaneous preterm birth and it can be offered to women with risk factors for preterm birth (evidence level II2B) according to the clinical practice guide by The Canadian Society of Gynecology in 2011. Reliability varies, according to the previous obstetric history, being a better predictor of preterm birth in women with a history of previous spontaneous preterm delivery (sensitivity $70 \%$ ) than in asymptomatic and low risk women (sensitivity $40 \%$ ). ${ }^{5}$ The gestational age at which the previous preterm birth occurred affects the frequency and rate of shortening in the current pregnancy (level of evidence II-2). The risk of premature labor increases as the length of the cervix decreases. Thus, if the cervix measures less than $15 \mathrm{~mm}$, the risk of premature delivery approaches $50 \%$. One of the hypotheses of cervical insufficiency is that it is due to a mechanical problem, hence the approach to address it has been cervical cerclage or with the pessary. ${ }^{6}$

\section{Cervical pessary: mechanism of action}

The cervical pessary is a silicone device designed to support the cervix. Its mechanism of action is not clearly established; it could achieve a reduction of the pressure of the uterus on the cervical canal through an alteration of the uterus-cervical angle so that the force of the weight of the uterine contents moves away from the internal cervical canal and is exerted on the back, towards the sacrum. It could also strengthen the immunological barrier between the choroamniotic extravascular space and the vaginal flora. It is an easy-touse, non-invasive, inexpensive tool that does not require anaesthesia and is potentially promising to reduce the risk of premature delivery in patients with risk factors. ${ }^{2}$ So it is a possible alternative to cerclage and/or progesterone treatments. It has been used for this purpose since 1950. At the beginning they were designed for genital prolapse and in the 1970s the first ones for use in pregnant women were developed. There are different types (Smith, Hodge, Risser, Hamann, Portex ), but the most recent studies use the Arabin pessary, with the advantage of a European certification (CEO482, MED / CERT I SO9003 / EN46003). It is a flexible silicone ring, available in several sizes. The external diameter varies between $65-70 \mathrm{~mm}$, the internal diameter between $32-35 \mathrm{~mm}$ and its height is $17-30 \mathrm{~mm}$. It is perforated to enable the flow of vaginal and cervical secretions. It is built in such a way that the caudal portion of the device surrounds the neck, so it has been said that it can provide an additional benefit by preventing cervical dilation, the deterioration of the mucous plug and the exposure of the membranes. It is associated with a low rate of major complications: only a slight discomfort and an increase of vaginal flow without an increase in the rate of vaginal infection.

\section{Use of the cervical pessary as a unique preventive treatment}

Until 2012, only prospective or retrospective observational studies with methodological biases had been published, so the 2010 Cochrane review agreed there was no evidence that pessaries reduced prematurity. ${ }^{8}$ From 2012 to December 2016, 6 large randomized 
controlled trials (RCTs) have been published. The intervention is pessary Arabin versus expectant treatment.

A) RCT in single asymptomatic pregnancies with cervix $\leq 25 \mathrm{~mm}$ measured by transvaginal echography between week $18-24^{+6}$ of pregnancy:

I. Multicenter Spanish study PECEP of 2012 with 385 women with cervix $\leq 25 \mathrm{~mm}$ between week $18-22$ of gestation in a not selected group of general population. ${ }^{7}$ A single pessary size of Arabin (25$65-32 \mathrm{~mm}$ ) was used. The obstetricians who participated carried out sonographic training in the placement of the pessary. The pessary was retired at 37 week. In case of premature rupture of membranes during the study, the pessary remained if there were no signs of chorioamnionitis. The primary outcome was spontaneous preterm labor before week 34 . This rate was significantly lower in the pessary group ( $6 \%$ vs $27 \%$ ), and also in gestations shorter than 28 and 37 weeks. The need for tocolysis or use of glucocorticoids was lower. Neonatal results (3\% vs 16\%) was also significantly better in the pessary group. The sole adverse effect was an increase in vaginal discharge. The limitation is that it is an open trial and that the rate of premature delivery in the expectant management group was $27 \%$, much higher than the one published previously. They conclude that the cervical pessary is an affordable alternative, safe and effective for the prevention of spontaneous preterm birth in a population of women adequately selected for cervical length of the second trimester.

II. RCT published by Hui in 2013 in single pregnancies of asymptomatic women with cervix $\leq 25 \mathrm{~mm}$ between week 20 24 with no history of cervical insufficiency or cerclage, in 108 patients. ${ }^{9}$ The primary outcome was the same as in the PECEP study but no significant differences were observed in the rate of spontaneous preterm birth before week 34 (9.4\% vs. 5.5). The results were discordant with the PECEP study that included populations at higher risk than Hui's Chinese study (19.5\% vs. $11 \%$ patients with a history of previous preterm birth).

III. European multicenter RCT of Nicolaides ${ }^{10}$ in 2016. 935 patients with cervix $\leq 25 \mathrm{~mm}$ between weeks $20-24^{+6}$ were recruited. Those having cervix $\leq 15 \mathrm{~mm}$ at the start or during the study, received also $200 \mathrm{mg}$ vaginal progesterone up to week $33^{+6}$ ( $45 \%$ of the total) regardless of whether they had a pessary or not. The primary outcome was the rate of spontaneous preterm birth before week 34 and there were not significant differences (12\% vs. $10.8 \%$ in the control group). The results also did not vary in the analysis by subgroups when adjusted for cervical length, use of progesterone or antibiotic treatment for vaginal infection or obstetric history. Secondary outcomes of neonatal and perinatal morbidity and mortality do not differ with the use of the pessary. However, there was a significant increase in vaginal discharge. The rate of spontaneous preterm birth before week 34 was $11 \%$, higher than the expected $6 \%$. It seems that there was a bias of participation in pregnant women with shortened cervix. The decision to use progesterone could mitigate the effect of the pessary in patients with cervix $<15 \mathrm{~mm}$ and its high rate of pessary withdrawal before week $34(24.5 \%)$ could explain the discordant results between this study by Nicolaides and the PECEP study.

B) Randomized controlled trials in multiple pregnancies

I. ProTwin ${ }^{11}$ Study in 2013 in 40 hospitals in the Netherlands, in a general population of asymptomatic multiple pregnancies $(98 \%$ twins and $2 \%$ triplets) regardless of cervical length, between weeks 16-22. 808 patients took part in the study. The primary outcome was deficient perinatal outcome and it did not differ significantly in both groups (13\% vs. $14 \%$ in the control group). Among the secondary results that were analysed, the rate of premature delivery before 37,32 and 28 weeks, was similar in both groups. An analysis of rheumatism by subgroups was made when the cervix was shorter than $38 \mathrm{~mm}$. In them the deficient perinatal result was lower in the pessary group in a significant way, also the median gestational age at delivery was higher and the pessary significantly reduced the risk of preterm birth in pregnancies below 28 and 32 weeks of gestation. They conclude that the use of the pessary is not relevant in an asymptomatic general population of multiple pregnancies but the subgroup of patients with cervix shorter than $38 \mathrm{~mm}$ should be featured in future studies. With the same database another multivariant analysis ${ }^{12}$ was carried out, looking for other characteristics, either maternal or having to do with the pregnancy at the beginning, that could improve the identification of women more likely to benefit from the placement of the pessary. The result was that a short cervix, monochorionicity and nulliparity were predictive factors of a benefit in the insertion of the pessary for an adverse perinatal outcome. They suggest that their multivariant model to identify women who would benefit most from the pessary is superior to a strategy based solely on cervical length. External validation is necessary in future studies in the area.

II. PECEP-TWINS study by Goya. ${ }^{13}$ RCT that includes 137 asymptomatic twin gestations in 5 hospitals in Spain, regardless of their obstetric history and with cervix $\leq 25 \mathrm{~mm}$ between $18-22$ weeks. The protocol was similar to the PECEP study. They also devised a new ultrasound technique for measuring the cervix with the pessary in situ. They did not remove the pessary if there was premature membrane rupture in the absence of chorioamnionitis. The rate of spontaneous preterm birth in gestations shorter than 34 weeks was significantly lower in the pessary group (16.2\% vs. $39.4 \%$ ). The cumulative percentage of patients who spontaneously gave birth before 34 weeks was significantly greater in the pessary group. The tocolysis and the glucocorticoids were used less, but the differences were not significant; there were no significant differences in neonatal morbidity and mortality. There were no significant maternal adverse effects, although there was a significantly higher rate of vaginal discharge. There were no differences in the rate of premature delivery before weeks 28 or 37. They conclude that in twin pregnancies selected by short cervix the placement of the pessary in week 22 reduces the rate of spontaneous premature delivery in gestations shorter than 34 weeks.

III. RCT by Nicolaides ${ }^{14}$ in 2016 carried out in 23 European hospitals, in an unselected general population of asymptomatic multiple pregnancies, regardless of the length of the cervix measured at week $20-24^{+6}$. It includes 1180 pregnant women. There were no significant differences in the rate of preterm delivery before 34 weeks ( $13.6 \%$ vs. $12.9 \%)$ or perinatal death $(3.4 \%$ vs. $30.7 \%)$, low weight $<2500 \mathrm{~g}(77.2 \%$ vs. $0.1 \% 69)$ or adverse neonatal event $(15.2 \%$ vs. $11.9 \%)$. Given that they were patients not selected for cervical length, the medians of that length at the time of randomization was $32 \mathrm{~mm}$; so the subgroup analysis with short cervix ( $\leq 25 \mathrm{~mm}$ which were only $18 \%$ of the sample) showed no benefit with the colocation of the pessary with a rate of premature birth in gestations shorter than 34 weeks (31.1\% vs. $25.9 \%)$. 
They conclude that, both for unselected twin populations and in the short cervix subgroup, the placement of the pessary does not reduce the rate of spontaneous preterm birth or improve the neonatal outcomes. The limitations of this study are that it is open and the researchers who placed the pessary did not receive training to do so (they did for the ultrasound). These three studies and well designed, but there is heterogeneity among them, which could explain the difference in their results. The PECEP-Twins trial refers to selected population with short cervix and twin pregnancies, whereas the results for twins with short cervix in the ProTwin and Nicolaides studies, came out from a subgroup analysis outside the original design. In the ProTwin trial the median length of the cervix was $44 \mathrm{~mm}$ (only $1 \%$ measured $<25 \mathrm{~mm}$ ), whereas in the Nicolaides study it was $32 \mathrm{~mm}$ and $18 \%$ with cervix $\leq 25 \mathrm{~mm}$. There are at the moment up to 9 multicenter RCT being carried out, one in France (Pesar-One PHR (2013)) in twin gestations with cervix $<35 \mathrm{~mm}$. Their results will help us to clarify whether or not there is scientific evidence for the use of the Arabin pessary in the prevention of premature birth.

\section{Use of the pessary as a single treatment compared or in combination with other methods}

Given that no isolated intervention has been definitively effective in the prevention of premature birth, studies have been designed with combined treatments:

\section{Progesterone versus pessary}

RCT of Karbasian, ${ }^{15}$ in 2016, in 144 single pregnancies with cervix $\leq 25 \mathrm{~mm}$ between $18-22$ weeks of pregnancy. $400 \mathrm{mg}$ vaginal progesterone daily are compared to the use of the pessary Arabin plus porgesterone, using 6 available sizes, according to the patient. The primary outcome was premature birth before 37 weeks and the secondary outcome premature delivery under 26,32 and 34 weeks, as well as low birth weight under $2500 \mathrm{~g}$, premature membrane rupture, chorioamnionitis or admission to the neonatal ICU. There were no significant differences in any of the results sought. The combined treatment had no additional benefit over vaginal progesterone alone to prevent premature delivery.

A. Pesapro ${ }^{16}$ study started in 2016. It is a Spanish open multicentre (31 hospitals) RCT for single pregnancies with short cervix with ultrasound at 19-22 weeks. It will compare $200 \mathrm{mg}$ vaginal progesterone daily versus Arabin pessary 65-25-32 mm until week $37^{+4}$ and 37 weeks respectively, to decrease the rate of spontaneous premature delivery before 34 weeks. It started in August 2012 and its results have not been published yet.

i. Quadruple P study of the Netherlands ${ }^{17}$ proposed in 2017. It is still underway and will compare $200 \mathrm{mg}$ vaginal progesterone and Arabin pessary, for the prevention of preterm birth in asymptomatic women with short cervix $\leq 35 \mathrm{~mm}$ between weeks $18-22$ in single pregnancies and in multiple pregnancies (not only twin gestations) between 16-22 weeks and short cervix $<38 \mathrm{~mm}$. The primary result will be the combined adverse outcome of perinatal mortality and morbidity. They also plan to conduct a subgroup analysis based on the length of the cervix $<25 \mathrm{~mm}$ vs $\geq 25 \mathrm{~mm}$, parity, previous preterm birth, chorionicity and number of fetuses.

\section{ii. Pessary versus cerclage}

The cerclage is effective but it is not free of risks. The pessary is easy, simple and safe. There is no evidence available to date. The PC study ${ }^{18}$ has been designed recently in the Netherlands. It is an open multicenter RCT, to compare cervical pessary versus cerclage in single pregnancies with short cervix and antecedent of preterm birth before 34 weeks, based either on the diagnosis of cervical insufficiency or previous preterm birth and cervix $\leq 25 \mathrm{~mm}$. The hypothesis is that the Arabin pessary will be as effective as cerclage. Women with primary indication of cerclage due to a diagnosis of cervical insufficiency and those with cervix $\leq 25 \mathrm{~mm}$ before 24 weeks who would be candidates for a secondary cerclage, are eligible to participate. They will be randomly assigned to receive cerclage or Arabin pessary. Both will be placed before 24 weeks or 16 weeks in case of primary intervention. They also plan to conduct a subgroup analysis based on primary or secondary indication of cerclage, number of previous preterm births, length of the cervix ${ }^{19}$.

\section{iii. Cerclage versus pessary versus vaginal progesterone}

Currently it is not possible to advise women at high risk of preterm birth and short cervix which of the three is the best treatment. In the retrospective study of Alfirevic ${ }^{13}$ in 2013 there were no differences in outcomes except a lower rate of preterm birth in pregnancies shorter than 34 weeks in the pessary group. It was necessary to design a randomized controlled trial, the open multicenter study SuPPoRT ${ }^{20}$ in the United Kingdom that will evaluate if the 3 interventions are equally effective to prevent premature birth in asymptomatic women with single pregnancies and short cervix $<25 \mathrm{~mm}$, randomized between 14 and $23^{+6}$ weeks. The primary outcome will be premature labor before week 37 . It will answer the dilemma of which is the most effective method. The women will be at high risk for having cervix $<25 \mathrm{~mm}$ and a previous history of pre- mature birth, premature rupture of the membrane before week 37 or conization history. The cerclage will be reserved at the time of randomization and the pessary will be removed in week $37.200 \mathrm{mg}$ vaginal progesterone will be administered daily until week 34 and the pessary will be withdrawn in week 37. An analysis of inflammatory biomarkers will also be performed before the intervention. The analysis of whether the response to the intervention is related to the previous biomarker status can help us identify subgroups of women who respond differently to each treatment and allow us to choose it more specifically for each woman.

\section{What evidence do the meta-analyses show us?}

The latest 3 meta-analyses published in 2017, pessary versus expectant management in the prevention of preterm labour are those of Saccone et al., ${ }^{21}$ in single gestations with short cervix, in twin gestations, ${ }^{22}$ and the one published by Jim et al. ${ }^{23 .}$

The meta-analysis of Saccone ${ }^{21}$ in single gestation with short cervix $\leq 25 \mathrm{~mm}$ includes 1420 women $\left(20-24^{+6}\right.$ weeks of gestation). They come from the analysis of previous 3 RCTs. ${ }^{7,9,10}$ In all of them the Arabin pessary was used. In two studies only the pessary was used as intervention (PECEP and Hui), and Nicolaides added 200mg vaginal progesterone up to week $33^{+6}$ if the cervix was $\leq 15 \mathrm{~mm}$. The primary outcome was the incidence of spontaneous preterm birth before week 34 and the secondary outcomes, among others, the spontaneous preterm delivery rate before weeks 37,32 and 28 , maternal secondary effects and neonatal outcomes. No result was statistically significant in favor of the use of the pessary except for a significantly higher risk of vaginal discharge. Although they planned the analyses by subgroups of patients according to whether they had 
had a previous spontaneous premature delivery or not, or different cutoff points according to the length of the cervix, this sub-analysis was not possible. They concluded with a level of evidence I that the data available up to date point to a lack of efficiency of the Arabin pessary in pregnancies with single fetus and short cervix. While this conclusion is in contrast with the Cochrane review ${ }^{24}$ of 2013, this review only includes one RTC, the 2012 PECEP. Therefore, the metaanalysis by Saccone is more reliable sice it includes all the RTCs available to date. This meta-analysis has limitations because none of the studies were double-blind; more than $50 \%$ of women came from a single study. ${ }^{10}$ In addition, there is a discordant rate of spontaneous premature birth (26.8\% in Goya, highly above the published rate) and the small number of RCTs did not allow a meta-analysis stratified by subgroups. Saccone analyzes the reasons why the pessary was more effective in the PECEP study, ${ }^{7}$ a specific training in the insertion of the pessary. Nicolaides ${ }^{10}$ included many centers, some with few cases and little training in the insertion of pessaries. Also, in this study (with the largest number of cases) there were more women with a previous spontaneous preterm birth in comparison with the PECEP study, ${ }^{7}$ so Saccone concludes that the pessary could be more effective in simple pregnancies with short cervix and without previous spontaneous premature delivery. To clarify this point further studies are needed. The meta-analysis of individual patients data will clarify whether the cervical pessary can be beneficial in subgroups such as single pregnancies with or without previous spontaneous premature birth, or at different cutoff points according to the length of the cervix.

Saccone meta-analysis in twin gestations. ${ }^{22}$ It includes the 3 RCTs published to date..$^{11,12,14}$ The methodology is identical to that of single gestations, with the same primary and secondary results. They also tried to conduct a subgroup analysis of twins with and witout previous spontaneous preterm birth and with cervix $\leq 25 \mathrm{~mm}$ and $\leq 20 \mathrm{~mm}$, which was not possible. 3 studies included twin gestations with and without previous spontaneous preterm birth and only the PECEPTWIN study included twin pregnancies with short cervix $\leq 25 \mathrm{~mm}$; while the other two RTCs included twin gestations not selected by the length of the cervix. Hence in the Saccone meta-analysis only the cases from these 3 studies that had short cervix were included. A total of 481 twin gestations. The use of the pessary in twin pregnancies with short cervix at 16-24 weeks was not associated with the prevention of spontaneous preterm birth before weeks $37,34,32$ and 28 . There were no differences in secondary outcomes, so we lack evidence to support the efficacy of the Arabin pessary in twin gestations with short cervix between weeks 16-24. The limitation of the meta-analysis is due to the availability of only $3 \mathrm{RCTs}$, which made it impossible to perform the analysis of sub groups and since the PECEP-TWIN concluded in the effectiveness of the pessary in twin with short cervix, the discrepancy of result is explained in the same way as in the meta-analysis of the single gestations, so the pessary could be effective in twin gestations with previous spontaneous preterm birth and short cervix whenever the results by subgroups could be analyzed.

The meta-analysis from Jim et al., ${ }^{23}$ includes 8 studies, the 6 RCTs published to date ${ }^{7-11,13,14}$ plus the 2003 Arabin study, ${ }^{25}$ and the $2016 \mathrm{Di}$ Tomasso $^{26}$ in twin gestations. The intervention is pessary to prevent premature births in risk pregnancies (short cervix, insufficiency, twins, etc) versus the control group with expectant or medical treatment. The comparison of gestational age at the time of delivery at $28,32,34$ and 37 weeks showed no significant differences, but the analysis of the mean gestational age at the time of delivery did reach significant differences. When comparing the use of tocolytics and corticoids, the use of the pessary reduced both rates significantly. No differences were observed regarding the risk of premature membrane rupture (4 of the 8 studies were included). Regarding neonatal outcomes, there was no statistical significance. In the analysis by subgroups of twin pregnancies or single pregnancies, in terms of gestational age at delivery and neonatal outcomes, there were no significant differences either. An analysis of the rate of premature birth in different gestational ages was performed. The heterogeneity in the results was due to the fact that the inclusion criteria of each study of the 8 was not identical in terms of gestational weeks, cervix length, parity, single or twin gestation, so the analysis was reduced to the subgroups of gestational age at the time of delivery at 28 and 34 weeks and showed that the pessary did not reduce preterm birth in these weeks. Therefore, this meta-analysis only concludes that the use of the cervical pessary can reduce the use of corticoids and tocolytics during the pregnancy. There were no differences in neonatal complications and the results did not improve in neither the analysis of twin or unique subgroups.

Jarde published two meta-analyses in July 2017: one on the prevention of preterm birth in twin pregnancies using progesterone, pessary or cerclage. ${ }^{27}$ It includes RCTs that use any of these strategies or the combination in asymptomatic twins. It includes 23 studies with 6626 patients. The primary outcomes were premature delivery rate before 34 and 37 weeks and neonatal mortality. None of the general interventions was beneficial for the prevention of preterm birth and its sequelae. Vaginal progesterone improved some important secondary outcomes in women in general and with short cervix but this author considers that additional research is required to reduce the risks of premature birth and its sequelae in twin pregnancies, including the use of combinations of therapies. In the other identical meta-analysis by Jarde but in single pregnancies ${ }^{28}$ (36 trials with 9425 patients), progesterone was the best intervention. The pessary showed an inconsistent benefit and the cerclage was not effective as a preventive measure.

\section{What evidence do the major revisions show us?}

The lastest Cochrane ${ }^{8}$ review from 2013 only includes the PECEP study. Despite the beneficial result in favor of the pessary according to this study, he concludes that more studies are needed in different settings and with different risk factors.The UPTODATE review $^{29}$ of May 2018 regarding premature birth, risk factors and reduction strategies: in unselected twin pregnancies, progesterone supplementation, use of the pessary, cerclage, bed rest or reduction of physical activity does not prolong pregnancy. In twin pregnancies with previous preterm birth or short cervix, the use of supplemental progesterone or a pessary is controversial. In single pregnancies, if short cervix $\leq 25 \mathrm{~mm}$ in week $18-24$ and there is no history of preterm delivery a pessary is not prescribed, as the body of evidence does not support its use to prolong pregnancy or improve neonatal outcome. Also the UPTODATE review of May 2018 in reference to cervical insufficiency ${ }^{4}$ considers the pessary an ineffective, not approved intervention. This is because the latest meta-analysis published do not support its use. The June 2018 review on measuring cervical length as a predictor, ${ }^{30}$ recommends not to carry out this test in twin gestations because no intervention has proven to be beneficial to prevent preterm birth in twin gestations with short cervix (GRADE 2C). For single pregnancies without premature labor and cervical $\leq 25 \mathrm{~mm}$ vaginal progesterone (GRADE 2C) is suggested. The cerclage does not 
prevent premature birth in this population and the pessary has not been adequately studied. The specific review of May 2018 regarding twin pregnancy ${ }^{29}$ considers the pessary an untested intervention in both unselected twins and those with cervix $\leq 25 \mathrm{~mm}$ and does not advise patients to use the pessary because no significant improvement has been shown in composite neonatal morbidity. No intervention has been shown to be effective in reducing the rate of premature birth in twins. ${ }^{31}$

\section{Recommendations of scientific societies}

a. The SOGC ${ }^{32}$ in its latest guide of 2011, regarding evaluation of cervical ultrasound length in the prediction of premature births, does not make a single reference to the pessary, and, on the contrary, it does to other preventive measures such as progesterone or cerclage. In asymptomatic and high risk women, due to the lack of proven effective interventions (without specifying what they are), the evaluation of routine cervical transvaginal length is not recommended (evidence level II-2E).

b. The $\mathrm{ACOG}^{33}$ in its 2017 guide to interventions on premature birth does not mention preventive strategies, it only refers to prenatal treatment with corticoids.

c. The US maternal-fetal medicine society $(\mathrm{SMFM})^{34}$ reviews in March 2017 the role of the cervical pessary in clinical practice. The FDA does not have a device approved as a cervical pessary for the prevention of premature birth, although it does have them approved with indications of genital prolapse. The Arabin pessary is not approved for sale in the US under any indication and can only be used within an RCT, so the guide concludes that at this time there is no conclusive evidence that the use of the cervical pessary, including that of Arabin, lowers the rate of premature delivery or improves maternal or fetal outcomes for women at high risk of preterm labor. It recommends that the placement of the cervical pessary in pregnancy be used only in the context of a clinical trial or research protocol.

d. The NICE Guideline of October 2016, on care and strategies in women with single pregnancy and risk or symptoms or signs of premature birth, considers vaginal progesterone or prophylactic cerclage (and does not refer to the pessary).

e. The 2017 Australian and New Zealand guide says that cervical evaluation between week $18-24$ is useful to identify women at higher risk of preterm birth who may benefit from interventions such as vaginal progesterone or cerclage, without naming the pessary.

f. The French guide of December 2016 recommends: in single asymptomatic pregnancy with no history or risk factors and short cervix between week 16-24 vaginal progesterone (GRADE B); Cerclage if history of at least 3 late spontaneous miscarriages or premature births (GRADE A). In single gestation, examination with transvaginal ultrasound between week 16-22 if there is a history of premature delivery before week 34 , to apply cerclage if cervix $<25 \mathrm{~mm}$ before 24 weeks (GRADE C). The pessary is not recommended for the prevention of preterm birth in a general population of asymptomatic women with twin pregnancy (GRADE A) and in populations of asymptomatic women with short cervix (professional advice).

\section{Conclusion}

Despite the initial expectations in favor of the use of the pessary as a non-invasive, cheap and effective method to prevent premature birth, to date, neither the best designed studies nor the meta-analyses that analyse them, support with scientific evidence the use of the Arabin pessary both in single pregnancies and in multiple gestations. The main scientific societies today do not include it as a preventive strategy in their clinical practice guidelines. Its use for the time being should be restricted to clinical trials, many of which are underway. The analysis of inflammatory biomarkers, in combination with the cervical length of the second term, as well as other risk factors and the analysis by subgroups of these patients in large multicentre trials, will allow us to define what is the real effectiveness of the Arabin pessary as a preventive strategy in preterm birth, alone or in combination with other strategies.

\section{Acknowledgments}

None.

\section{Conflict of interest}

The author declares there is no conflict of interest.

\section{References}

1. Chang HH, Larson J, Blencowe H, et al. Preventing preterm births: analysis of trends and potential reductions with interventions in 39 countries with very high human development index. Lancet. 2013;381(9862):223-234.

2. Keelan JA, Newnham JP. Recent advances in the prevention of preterm birth. F1000Res. 2017;1:6.

3. Iams JD, Goldenberg RL, Meis PJ et al. The length of the cervix and the risk of spontaneous premature delivery. N Engl J Med. 1996;334 (9):567572.

4. Berghella V. Cervical insufficiency. 2018.

5. Markham KB, Iams JD. Measuring the Cervical Length. Clin Obstet Gynecol. 2016;59(2):252-263.

6. Boelig RC, Bergella V. Current options for mechanical prevention of preterm birth. Semin Perinatol. 2017;41(8):452-460.

7. Goya M, Pratcorona L, Merced C, et al. Pesario Cervical para Evitar Prematuridad (PECEP) Trial Group. Cervical pessary in pregnant women with a short cervix (PECEP): an open-label randomised controlled trial. Lancet. 2012;379:1800-1806.

8. Abdel-Aleem H, Shaaban OM, Abdel- Aleem MA. Cervical pessary for preventing preterm birth. Cochrane Database Syst Rev. 2013;5:CD007873.

9. Hui SY, Chor CM, Lau TK, et al. Cerclage pessary for preventing preterm birth in women with a singleton pregnancy and a short cervix at 20 to 24 weeks: a randomized controlled trial. Am J Perinatol. 2013;30:83-288.

10. Nicolaides KH, Syngelaki A, Poon LC, et al. A randomized trial of a cervical pessary to prevent preterm singleton birth. $N$ Engl $J$ Med. 2016;374:1044-1052.

11. Liem S, Schuit E, Hegeman M, et al. Cervical pessaries for prevention of preterm birth in women with a multiple pregnancy (ProTWIN): a multicentre, open-label randomised controlled trial. Lancet. 2013;382 (9901):1341-1349.

12. Tajik PP, Monfrance MM, van 't Hooft JJ, et al. A multivariable model to guide the decision for pessary placement to prevent preterm birth in women with a multiple pregnancy: a secondary analysis of the ProTWIN 
trial. Ultrasound Obstet Gynecol. 2016;48(1):48-55.

13. Goya M, de la Calle M, Pratcorona L, et al. Cervical pessary to prevent preterm birth in women with twin gestation and sonographic short cervix: a multicenter randomized controlled trial (PECEP-twins). Am J Obstet Gynecol. 2016;214:145-152.

14. Nicolaides KH, Syngelaki A, Poon LC, et al. Cervical pessary placement for prevention of preterm birth in unselected twin pregnancies: a randomized controlled trial. Am J Obstet Gynecol. 2016;214(1):1-9.

15. Karbasian N, Sheikh M, Pirjani R, et al. Combined treatment with cervical pessary and vaginal progesterone for the prevention of preterm birth: A randomized clinical trial. J Obstet Gynaecol Res. 2016;42(12):1673-1679.

16. Cabrera-García L, Cruz-Melguizo S, Ruiz- Antorán B, et al. Evaluation of two treat ment strategies for the prevention of preterm birth in women identified as at risk by ultrasound (PESAPRO Trial): study protocol for a randomized controlled trial. Trials. 2015;16:427.

17. van Zijl MD, Koullali B, Naaktgeboren CA, et al. Pessary or Progesterone to Prevent Preterm delivery in women with short cervical length: the Quadruple P randomised controlled trial. BMC Pregnancy Childbirth. 2017;4;17(1):284.

18. Koullali B, van Kempen LEM, van Zijl MD, et al. A multi-centre, noninferiority, randomised controlled trial to compare a cervical pessary with a cervical cerclage in the prevention of preterm delivery in women with short cervical length and a history of preterm birth-PC. BMC Pregnancy Childbirth study. 2017;6;17(1):215.

19. Alfirevic Z, Owen J, Carreras E, et al. Vaginal progesterone, cerclage or cervical pessary for preventing preterm birth in asymptomatic singleton pregnant women with history of preterm birth and a sonographic short cervix. Ultrasound Obstet Gynecol. 2013;41:146-151.

20. Hezelgrave NL, Watson HA, Ridout A, et al. Rationale and design of SuPPoRT: a multi-centre randomised controlled trial to compare three treatments: cervical cerclage, cervical pessary and vaginal progesterone, for the prevention of preterm birth in women who develop a short cervix. BMC Pregnancy Childbirth. 2016;21;16(1):358

21. Saccone G, Ciardulli A, Xodo S, et al. Cervical Pessary for Preventing Preterm Birth in Singleton Pregnancies With Short CervicalLength : A Systematic Review and Meta-analysis. J Ultrasound Med. 2017;36(8):1535-1543
22. Saccone G, Ciardulli A, Xodo S, et al. Cervical pessary for preventing preterm birth in twin pregnancies with short cervical length: a systematic review and meta- analy sis. J Matern Fetal Neonatal Med. 2017;30(24):2918-2925.

23. Jin Z, Chen L, Qiao D, et al. Cervical pessary for preventing preterm birth: a meta-analysis. J Matern Fetal Neonatal Med. 2017;20:1-7.

24. Di Tommaso M, Seravalli V, Arduino S, et al. Arabin cervical pessary to prevent preterm birth in twin pregnancies with short cervix. $J$ Obstet Gynaecol. 2016;36:715-718.

1. Jarde A, Lutsiv O, Park CK, et al. Preterm birth prevention in twin pregnancies with progesterone, pessary, or cerclage: a systematic review and meta-analysis. BJOG. 2017;124(8):1163-1173.

25. Jarde A, Lutsiv O, Park CK, et al. Effectiveness and pessary for preventing preterm birth in singleton pregnancies: a systematic review and network meta-analysis. BJOG. 2017;124(8):1176-1189.

26. Julian N Robinson, Errol R Norwitz. Preterm birth: Risk factors and interventions for risk reduction. 2017.

27. Bergella V. Second trimester evaluation of cervical length for prediction of spontaneous preterm birth in singleton gestations. 2018 .

28. Stephen T, ChasenMD, Frank A. Twin pregnancy: prenatal issues. 2018

29. Lim K, Butt K, Crane J. Ultrasonographic cervical length assessment in predicting preterm birth in singleton pregnancies. J Obstet Gynaecol Can. 2011;33:486-499.

30. ACOG Practice Bulletin No 560. Medicalli indicated late-preterm and early-term deliveries. Obstet Gynecol. 2013;121:908-910.

31. McIntosh J, Feltovich H, Berghella V, et al. The role of routine cervical length screening in selected high- and low-risk women for preterm birth prevention. Am J Obstet Gynecol. 2016;215(3):B2-B7.

32. NICE guideline: preterm labour and birth. 2016.

33. RANZCOG College Statement $\mathrm{C}-\mathrm{Obs}$ 27. Measurement of cervical length for prediction of preterm birth. 2017.

34. Sentilhes L, Sénat MV, Ancel PY, et al. Prevention of spontaneous preterm birth: Guidelines for clinical practice from the French College of Gynaecologists and Obstetricians (CNGOF). J Obstet Gynecol Biol Reprod. 2016;45(10):1446-1456. 